\title{
Les relations de pouvoir comme fondement des stratégies d'intégration des usagers dans le design process
}

\author{
Rihab Zaidi \\ Doctorante et chargée de cours à l'Université Bordeaux Montaigne (MICA)
}

\begin{abstract}
Résumé
Cet article a pour objectif de démontrer l'importance de la prise en compte des relations de pouvoir dans la mise en place des stratégies d'intégration de l'usager. Pour ce faire, nous allons supposer que la stratégie d'intégration de l'usager est une « fonction stratégique » du dispositif du design axée sur des relations de pouvoir. Puis, nous allons problématiser le dispositif du design à partir du rapport « pouvoir-savoir » d'usage en mobilisant la modalité compétence / performance. Notre argumentation sera basée sur la méthodologie de travail du LUPI (Laboratoire des Usages et des Pratiques Innovantes) de la cité du design à Saint-Étienne.
\end{abstract}

\section{Mots-clés}

Les stratégies d'intégration de l'usager- les relations de pouvoir- dispositif - « pouvoir-savoir » d'usage- la modalité compétence / performance

\begin{abstract}
Power's relationships as the basis of the user's integration strategies in the design process - The current paper aims to show the importance of considering the power relations in the implementation of the user's integration strategies. In order to do this, we first suppose that the user's integration strategy is a "strategical function" of design device that focuses on the power relations. We would proceed secondly to problematizing the design device on the basis of the relation between "power \& knowledge" of use, which will be examined through the competence/performance modality. Our argumentation will be based on the methodology of LIUP's study. LIUP is The Laboratory of Innovative Usages and Practices, in the Cité du design in saint-Etienne.
\end{abstract}

\section{Keywords}

User's integration strategies- the power relations- device -power \& knowledge of usecompetence/performance modality

\section{Sommaire / Content}

o. Introduction

1. Le projet design : un dispositif régi par des relations de pouvoir

2. La modalité compétence/performance : une ébauche pour repenser la stratégie d'intégration des usagers dans le dispositif design

3. Conclusion

Bibliographie 


\section{Ocula ${ }^{20}$}

La place de l'usager en design

R. Zaidi I Les relations de pouvoir I DOI: 10.12977/ocula2019-8

\section{o. Introduction}

Il nous semble que la connaissance des stratégies d'intégration de l'usager dans la conception en design ainsi que la modélisation des figures de l'usager, dépassent la simple alternative d'une pratique du projet attentive ou non à l'usager. Nous pensons qu'avant de faire appel à l'usager, ou d'essayer de le rendre présent, le designer doit disposer d'une connaissance des relations de pouvoir qui se trament dans le design process. Celles-ci vont rendre visibles le pouvoir et le savoir de l'usager pour qu'il soit à l'origine d'une « approche usage ». Quel serait donc l'intérêt d'une telle démarche dans la mise en place d'une stratégie d'intégration de l'usager?

Pour répondre à cette question, nous allons organiser notre réflexion en deux parties. Nous allons tenter de rendre compte, dans un premier temps, de la dynamique de la logique projectuelle du design afin de supposer que la stratégie d'intégration de l'usager est une « fonction stratégique » du dispositif du design axée sur des relations de pouvoir. Nous exposons, dans un second temps, une piste expérimentale pour penser la stratégie d’intégration des usagers dans le dispositif du design à partir du rapport « pouvoir-savoir » d'usage qui sera questionné à partir de la modalité compétence / performance. Un exemple viendra illustrer notre argumentation. Nous nous appuyons sur la méthodologie de travail du LUPI (Laboratoire des Usages et des Pratiques Innovantes) de la cité du design à Saint-Etienne. Ceci nous permet d’interroger l'impact de la diversité des compétences sur la stratégie d'intégration de l'usager.

\section{Le projet design : un dispositif régi par des relations de pouvoir}

Nous partons du postulat que le design est une discipline du projet. Or, contrairement aux autres régimes de la conception, le processus de conception en design est doté d'une pensée dynamique qui lui permet de surpasser la logique séquentielle propre à la pratique classique de la gestion de projet. D'où notre volonté de penser la logique projectuelle (cf. Vial 2014) du design en tant que dispositif. Pour mieux nous faire comprendre, il convient d'expliciter davantage le sens du terme dispositif en nous référant à Giorgio Agamben. Ce dernier s'est basé sur les trois critères essentiels du dispositif révélés par Foucault dans un entretien réalisé en 1977 et qui sont les suivants :

- « il s'agit d'un ensemble hétérogène qui inclut virtuellement chaque chose, qu'elle soit discursive ou non » (Agamben 2007 : 10).

- « le dispositif a toujours une fonction stratégique concrète et s'inscrit toujours dans une relation de pouvoir »(Agamben 2007 : 10).

- « il résulte du croisement des relations de pouvoir et de savoir » (Agamben 2007 : 11).

En reprenant ces trois critères, Agamben situe le terme dispositif dans un contexte plus général. Il le définit de la manière suivante : « j’appelle dis- 


\section{Ocula ${ }^{20}$}

La place de l'usager en design

R. Zaidi I Les relations de pouvoir I DOI: 10.12977/ocula2019-8

positif tout ce qui a, d'une manière ou d'une autre, la capacité de capturer, d'orienter, de déterminer, d'intercepter, de modeler, de contrôler et d'assurer les gestes, les conduites, les opinions et les discours des êtres vivants » (Agamben $2007:$ 31). En ce sens, nous pouvons supposer que la logique projectuelle du design qui touche désormais à tout l'écosystème de notre quotidien (produits, service, expérience, espace, interface, etc.) fonctionne comme un dispositif.

Ce qui nous intéresse le plus, dans un premier temps, c'est le fait que le dispositif est doté d'une "fonction stratégique » toujours rapportée à des relations de pouvoir. Nous rappelons que nous nous proposons ici de nous intéresser à une fonction stratégique bien déterminée, ce qui correspond à la stratégie d'intégration de l'usager. A partir de là, nous pouvons considérer la stratégie d'intégration de l'usager comme une « fonction stratégique » du dispositif du design. Elle s'inscrit dans l'ensemble des relations de pouvoir mobilisées par le projet. Mais qu'entendons-nous au juste par le terme pouvoir?

Pour éviter les amalgames que ce mot peut induire, une précision terminologique s'impose. Nous nous référons encore une fois à la pensée de Foucault pour expliciter le sens du terme pouvoir. En effet, le pouvoir selon cet auteur n'est pas une relation entre dominant et dominé. Il ne peut pas non plus être identifié et localisé en un seul point (institution, appareil étatique, etc.). Foucault défend l'idée d'une " omniprésence du pouvoir ». Il démontre que le pouvoir signifie une " multiplicité des rapports de force qui sont immanents au domaine où ils s'exercent, et sont constitutifs de leur organisation » (Foucault 1994 : 121-122).

La multiplicité des rapports de forces engage la multiplicité des acteurs. Bien entendu, il serait sans doute vain de démontrer que le dispositif du design est pluri-acteurs et que cette pluralité est fondamentale. Ainsi, nous pouvons déduire, dans un premier temps, que la stratégie d'intégration de l'usager est une « fonction stratégique » assumée collectivement dans le dispositif design et régie par les relations de pouvoir propres à l'écosystème du projet. La structuration de cette stratégie va donc dépendre de l'identification et de la gestion des rapports de forces durant les différentes phases du design process. À quelle condition la stratégie d'intégration de l'usager sera-t-elle donc possible?

Nous pensons qu'avant de faire appel aux usagers, ou d'essayer de les rendre présents dans le dispositif, une connaissance approfondie des usages est nécessaire. Autrement dit, il vaut mieux essayer d'expérimenter et d'observer les usages par rapport à l'ensemble de leurs écosystèmes, avant d'essayer d'identifier l'usager-cible en rapport direct avec les premières intentions stratégiques du projet. Analyser les usages revient plutôt à engager une « approche usage » en vue de la mise en place de la stratégie d'intégration de l'usager dans le dispositif du design.

Dès lors, une autre précision terminologique s'impose afin de lever l'ambiguïté du terme usage. Selon Arendt, l'usage donne « à l'artifice humain la stabilité, la solidité qui, seules, lui permettent d'héberger cette instable et mortelle créature, l'homme » (Arendt 1988 : 187). Arendt démontre que l'usage 


\section{Ocula ${ }^{20}$}

La place de l'usager en design

R. Zaidi I Les relations de pouvoir I DOI: 10.12977/ocula2019-8

ne peut user que la durabilité. Ainsi, elle distingue l'usage de la consommation conçue comme l'" action d'amener quelque chose à son terme ${ }^{1}$ ». Cela n'empêche pas l'usage, selon elle, de contenir « certainement un élément de consommation, dans la mesure où le processus d'usure a lieu par contact entre l'objet et l'organisme vivant qui consomme...la destruction, encore qu'inévitable, est incidente à l'usage, mais inhérente à la consommation » (Arendt 1988 : 189). Michel de Certeau (1990) est du même avis. Selon lui, l'usage est une «fabrication » issue de la consommation.

Nous pouvons notamment distinguer l'usage de l'utilisation, car utiliser signifie " tirer parti de (quelque chose), faire servir (quelque chose) à une fin déterminée ${ }^{2}{ }^{2}$. C'est ce que Josiane Jouët démontre en affirmant que la « construction de l'usage ne se réduit dès lors pas aux seules formes d'utilisation prescrites par la technique qui font certes partie de l'usage, mais s'étend aux multiples processus d'intermédiations qui se jouent pour lui donner sa qualité d'usage social. » (Jouët 2000 : 499).

L'usage exprime aussi une volonté de " faire agir (un objet, une matière), pour obtenir un effet ${ }^{3} »$. Il est aussi moins impératif que l'utilisation et la consommation (cf. Grawitz 1991). Mais il comprend quand même « l'ensemble des règles et des pratiques qui régissent les rapports sociaux et qui sont les plus couramment observées ${ }^{4}$ ». L'usage s'exprime aussi dans la durée, l'ancienneté ou la fréquence qui assurent le passage de l'usage vers une "pratique sociale" » (cf. Akoun 1999). Ceci semble s'écarter de la définition que Stiegler attribue à l'usage. Selon lui, « qui dit usage, dit usure, c'est-à-dire aussi jetabilité » (Stiegler $2004: 244$ ). Ainsi, il oppose usage et pratique et ne les pense pas dans la continuité.

Essayons donc de récapituler. Nous associons l'usage à l'instar d'Arendt à la stabilité, solidité et durabilité de l'action menée par l'usager. L'usage transcende notamment la consommation et l'utilisation mais ne rompt pas avec eux. Par conséquent, une stratégie d'intégration de l'usager centrée sur une approche par l'usage doit être extrêmement sensible et ouverte à tous les signaux forts et faibles de l'usage. Cette ouverture du champ focal va permettre au designer d'aller au-delà de la maîtrise du besoin. Elle l'invite à s'immerger à l'intérieur de la complexité de l'usage, à saisir ses différents enjeux et à construire l'écosystème du projet à partir des divers signaux identifiés. L'intégration de l'usager doit être donc pensée et réalisée en fonction de cet écosystème.

Afin de mieux saisir les enjeux de la stratégie d'intégration de l'usager, il faut d'emblée accepter le fait qu'il s'agisse d'une action collective. Or, toute action collective loin d'être « un exercice gratuit » (Crozier et Friedberg 2014 : 23) est prédéfinie par une « réflexion en termes d'objectifs » (Crozier et Frie-

1 Centre national de ressources textuelles et lexical. En ligne. Page consultée le 19 février 2018. Disponibilité sur : <http://cnrtl.fr/definition/consommation>.

2 Centre national de ressources textuelles et lexical. En ligne. Page consultée le 19 février 2018. Disponibilité sur : <http://cnrtl.fr/definition/utiliser>.

3 Entrée « usage » du dictionnaire Le petit Robert

4 Centre national de ressources textuelles et lexical. En ligne. Page consultée le 19 février 2018. Disponibilité sur : <http://cnrtl.fr/definition/usage>. 


\section{Ocula ${ }^{20}$}

La place de l'usager en design

R. Zaidi I Les relations de pouvoir I DOI: 10.12977/ocula2019-8

dberg 2014 : 57). D'où le risque qu'elle soit « entièrement déterminée par les catégories de la fin et des moyens » (Arendt 1988 : 194-195). Dans ce cas-là, l'usager est, à la fois la finalité de la stratégie d'intégration et un moyen pour l'atteindre. Cette logique « strictement utilitaire » induit, la plupart du temps, une vision marchande et mercantile de l'usage. Sur ce point, il semble important de préciser que cette logique utilitaire va à l'encontre du concept de stratégie. Ce concept désigne ici un processus qui amène un dépassement et permet de le rendre possible comme l'ont montré Crozier et Friedberg. En quoi consiste ce dépassement visé par la stratégie d'intégration de l'usager? Comment le designer peut-il l'actualiser en pensant le dispositif design à travers les rapports de pouvoir?

Pour apporter quelques éléments de réponses à cette question, nous admettons à partir de la vision foucalidienne que « le pouvoir produit du savoir (et pas simplement en le favorisant parce qu'il le sert ou en l'appliquant parce qu'il est utile) » (Foucault $1993:$ 36). Ceci nous permettra de supposer que le savoir, et plus précisément le savoir d'usage, est l'objectif principal du dépassement de la stratégie d'intégration de l'usager.

Ce savoir doit être, d'une part, à l'origine de la mise en place de la stratégie d'intégration. En effet, le designer doit disposer d'un savoir concernant les méthodes et les outils nécessaires lui permettant d'assurer l'analyse et l'observation des usages pour identifier et intégrer les usagers. D'autre part, ce savoir ne doit pas être figé. Le designer doit ajuster ses outils au contexte d'usage et à l'ensemble des rapports de forces du dispositif du design. De plus, il doit aborder, à chaque fois, cette stratégie d'intégration comme une nouvelle opportunité de savoir.

Par conséquent, nous estimons que la stratégie d'intégration de l'usager doit être interrogée à travers le rapport « pouvoir-savoir » d'usage. Nous jugeons que cette manière de penser la stratégie d'intégration nous permet de dépasser la vision instrumentaliste de l'usager. Elle nous évite de le considérer comme une fin et un moyen du processus de conception, voire comme un " sujet de connaissance qui produirait un savoir, utile ou résistif au pouvoir » (Foucault $1993: 36$ ). La stratégie d'intégration considère l'usager comme un sujet de connaissance apte à produire un savoir et des modalités de connaissance de l'usage dans le dispositif du design régi par des relations de pouvoir. Autrement dit, il faut que la stratégie d'intégration de l'usager apporte de nouveaux savoirs concernant l'usage à tous les acteurs du projet, y compris l'usager qui est considéré la plupart du temps comme une source première de connaissance sur l'usage. Ceci implique une revalorisation et une prise en considération du pouvoir d'agir de l'usager dans la stratégie de son intégration.

Par ailleurs, penser la stratégie d'intégration de l'usager, dans le dispositif du design à travers le rapport « pouvoir-savoir » d'usage, revient à déterminer « les formes et les domaines possibles de la connaissance» d'usage (Foucault 1993 : 36). Cette connaissance concerne surtout les compétences acquises lors de la performance du dispositif du design. Or, selon Greimas (1976), la performance présuppose la compétence mais non inversement. 


\section{Ocula ${ }^{20}$}

La place de l'usager en design

R. Zaidi I Les relations de pouvoir I DOI: 10.12977/ocula2019-8

\section{La modalité compétence/performance : une ébauche pour repenser la stratégie d'intégration des usagers dans le dispositif design}

Pour rendre nos propos plus concrets, nous souhaitons nous référer à l'exemple de la méthodologie du LUPI. Il s'agit d'une méthodologie prospective axée sur l'exploration et les études des usages ${ }^{5}$ qui vise à accompagner des entreprises dans la définition de leurs stratégies par l'immersion dans un contexte d'usage. Cette méthodologie s'étale sur trois phases. Une première phase concerne le cadrage. Elle vise à favoriser l'échange entre l'équipe du LUPI et les représentants de l'entreprise. Le but de cette première phase consiste à cerner la problématique et identifier les profils des usagers à rencontrer. Une deuxième phase d'observation in situ s'ensuit. Durant cette phase, les participants seront amenés à observer les usagers. Un guide d'entretien conçu par les designers chargés du projet est mis à leur disposition pour qu'ils puissent accomplir cette mission. Une troisième phase de restitution va permettre aux participants de partager leurs retours d'expériences sur le déroulement des rencontres avec les usagers et recueillir l'ensemble des informations. Ceci permettra aux designers de lancer une séance de créativité pour co-concevoir avec les participants des scénarios innovants qui servent à lancer la phase d'idéation du projet.

Par soucis de clarté, nous souhaitons nous référer brièvement à l'exemple du LUPI de la « convivialité dans l'espace public ». Nous avons mené une observation participante dans ce projet qui consiste à questionner le rapport des personnes âgées à l'espace public via l'usage. Son but consiste à améliorer et à favoriser l'appropriation de l'espace public par les personnes âgées. Les participants ont été sélectionnés en deux temps.

L'équipe projet composée essentiellement de trois designers dont un designer manager et le coordonnateur LUPI, convoque, dans un premier temps, des participants qui sont directement concernés par la commande du projet. Il s'agit : d'une présidente d'un groupe de parole personnes âgée ; d'un chargé de projets en politique gérontologique à domicile, pôle gérontologie et de l'autonomie ; d'une chef de projet, coordonnatrice en charge du développement ; de deux directeurs généraux d'entreprises ; du directeur d'une maison de quartier ; d'une directrice adjointe d'un centre social de Beaulieu ; d'un président d'un espace retraités ; d'un président, trésorier d'une association. Suite à la phase de cadrage, l'équipe projet et les participants que nous venons de citer vont identifier des profils à rencontrer.

Il convient de signaler que l'identification de ces participants a été basée sur un rapport d'expérimentation fondé sur une approche par l'usage. Il s'agit plus précisément de l'expérimentation de la mise en place d'un dispositif composé d'une dizaine de chaises en libre service dans des jardins publics. Cette expérimentation a permis aux designers d'identifier les acteurs qui ont été directement impliqués dans le projet. Sinon, il s'agit aussi des acteurs qui seront liés

5 Cité du design. En ligne. Page consultée le 10 janvier 2018. Disponibilité sur : <https://www.citedudesign.com/fr/entreprises/271014-laboratoire-des-usages-etdes-pratiques-innovantes $>$. 


\section{Ocula ${ }^{20}$}

La place de l'usager en design

R. Zaidi I Les relations de pouvoir I DOI: 10.12977/ocula2019-8

de près ou de loin à la mise en place et à l'expérimentation du dispositif. Les rencontres se font en binômes et les profils identifiés sont les suivants : personne âgée qui n'a pas envie de se socialiser ; services municipaux ; bureau de contrôle ; juriste, adolescents (groupe) ; enfant / parent ; éducateur spécialisé / médiateur adolescence / médiateur vieillesse, commerçant / exploitant de l'espace public / bouquiniste / bibliobus ; personnes résidentes stéphanoises issues de l'immigration ; association handicap, architectes / artistes... collectif qui investit l'espace public, élu / adjoint à l'urbanisme (VSE), docteur en architecture.

Un premier constat s'impose : la diversité des acteurs signifie que nous sommes face à une diversité de compétences. En effet, Crozier et Friedberg ont souligné que « les acteurs sont inégaux devant les incertitudes pertinentes du problème. Ceux qui par leur situation, leurs ressources ou leurs capacités ... sont capables de les contrôler, utiliseront leur pouvoir pour s'imposer face aux autres » (Crozier et Friedberg 2014 : 24). Autrement dit, les acteurs seront inégaux les uns par rapport aux autres au regard de leurs compétences. Ainsi, leurs appréhensions et leurs implications dans la résolution de la problématique traitée par le dispositif du design seront inégales.

Ayant participé et animé un certain nombre d'ateliers participatifs, nous pouvons partir d'un constat empirique de l'impact de l'« incertitude » évoquée par Crozier et Friedberg sur les relations entre les différents acteurs du dispositif et sur le dispositif design lui-même. Nous précisons que son impact concerne surtout la participation des acteurs. En effet, certains acteurs ne participent pas au même titre que les autres, certains ont plus de difficultés dans la prise de parole pour exprimer un point de vue, alors que d'autres monopolisent la parole. Disons le plus clairement, c'est le manque de compétences par rapport à la problématique du projet qui risque de créer un déséquilibre dans la stratégie d'intégration de l'usager, et d'impacter sur la performance du dispositif du design dés l'amont du projet. Ce problème persiste même si le designer-animateur-facilitateur va constamment essayer de synthétiser, structurer, introduire, réintroduire la problématique, et veiller à ce que tous les acteurs s'expriment et participent dans les échanges et la définition de la problématique à parts égales.

Dans l'exemple du LUPI, la veille se fait après la phase de cadrage. Or, il nous semble que, dans cette phase, les acteurs auraient pu bénéficier d'un accès plus large à la documentation de la veille. En un certain sens, la veille aurait pu augmenter leurs compétences et par conséquent leurs performances dans la définition de la problématique et l'identification des usagers à rencontrer. A cet effet, nous suggérons une piste expérimentale qui va traiter le paramètre de la compétence dans le dispositif du design et plus précisément dans la stratégie d'intégration de l'usager. Son objectif consiste à repenser cette stratégie à partir du rapport « pouvoir-savoir » d'usage qui sera questionné via la modalité compétence - performance.

Il nous semble donc opportun que l'équipe projet procède ainsi :

- Elle commence par construire un référentiel de compétences. Celui-ci permettra à l'équipe projet de fixer les compétences requises pour l'amont et l'aval de la stratégie d'intégration de l'usager dans le dispositif design. 


\section{Ocula ${ }^{20}$}

La place de l'usager en design

R. Zaidi I Les relations de pouvoir I DOI: 10.12977/ocula2019-8

- Elle effectue par la suite des entretiens individuels ou des questionnaires pour faire une évaluation approximative des niveaux de compétence de l'ensemble des acteurs (participants et usagers) en amont de la performance.

- Pour analyser l'impact de ces compétences sur la stratégie d'intégration de l'usager à travers le rapport pouvoir-savoir d'usage, il nous semble opportun de « construire d'abord le système complet des interactions qui se nouent entre ses différents acteurs » (Mucchielli 1997 : 26). Il s'agit d'expliciter et de représenter les grandes catégories d'échange que les acteurs sont susceptibles d'avoir entre eux pour identifier la problématique (cf. Mucchielli 1997).

- Par la suite, il nous semble opportun d'essayer de repérer, à partir de ces grandes catégories, les relations qui ont un impact $(+)$ et les relations qui ont un impact $(-)$ sur l'intégration des usagers dans la phase de cadrage de la problématique. Les relations qui ont un impact (+) sont celles qui, malgré la diversité des compétences de leurs acteurs, semblent capables de faire évoluer la performance collective du dispositif à partir du rapport pouvoir-savoir d'usage. En effet, si l'acteur (A) dispose de plus de compétence que l'acteur (B) par rapport à la problématique traitée, l'acteur (A) risque de dominer l'acteur (B) dans l'ensemble des interactions qui vont avoir lieu. D'où le fait que l'acteur (B) sera moins intégré que l'acteur (A) dans le dispositif du design. La relation (AB) peut donc avoir un impact (+) si l'écart entre (A) et (B) n'est pas flagrant. Dans ce cas-là, (B) peut acquérir les compétences requises en échangeant avec (A). Mais la relation (AB) a un impact $(-)$ si l'écart de compétences entre A et B est important. Par ailleurs, il faut que (B) acquiert des compétences avant la mise en relation avec (A).

- Dernière étape, celle de l'ajustement qui sera assuré dès l'amont du projet par le dispositif design. Nous allons essayer d'équilibrer l'impact (-) de la relation (AB) en augmentant les compétences de (B) par rapport à (A) mais surtout par rapport à la problématique traitée. Nous allons anticiper les déséquilibres et prévoir des ajustements de compétences pour assurer le bon déroulement de la performance du dispositif design.

\section{Conclusion}

Nous souhaitons signaler que nous ne proposons pas de formater les compétences et l'intentionnalité des usagers. Nous ne tentons pas non plus de normaliser l'action pour la contenir et la programmer. Notre proposition envisage surtout d'aborder la stratégie d'intégration de l'usager par le biais du rapport « pouvoir-savoir » d'usage afin de valoriser la prise en considération des rapports de force dans le dispositif du design, pour augmenter le pouvoir d'agir de l'usager en prenant en considération ses compétences initiales concernant la thématique du projet. Ceci va à l'encontre de toutes « conceptions statiques » et séquentielles de l'usage car nous défendons l'idée d'un usager qui a déjà des compétences qui, lorsqu'il affronte des épreuves, se transforment en performances. C'est ce qui lui permet d'accomplir des actions au cours du dispositif du design et d'augmenter son pouvoir d'agir. 


\section{Ocula ${ }^{20}$}

La place de l'usager en design

R. Zaidi I Les relations de pouvoir I DOI: 10.12977/ocula2019-8

D'où l'intérêt d'interroger la programmation de la stratégie d'intégration via la dynamique des relations de pouvoir afin de prévoir des ajustements qui peuvent préserver le pouvoir d'agir de l'usager. Ceci est surtout dû au fait qu' " aucune procédure, même parfaitement programmée, n'échappe à des accommodations qui peuvent aussi bien emprunter aux routines acquises que promouvoir des innovations » comme l'a bien démontré Jacques Fontanille (2010 : 9).

\section{Bibliographie}

Agamben, Giorgio

2006 Qu'est-ce qu'un dispositif? ; trad.fr par M. Rueff, Paris, Rivages poche, 2007.

Akoun, André (dir.)

1999 Dictionnaire de sociologie, Paris, le Robert.

Arendt, Hannah

1958 Condition de l'homme moderne, trad. Fr. par G. Fradier, Paris, Pocket, 1988.

Crozier, Michel ; Friedberg, Erhard

2014 L'acteur et le système, Paris, Seuil.

De Certeau, Michel

1980 L'invention du quotidien, 1. Arts de faire, Paris, Gallimard.

Fontanille, Jacques

2010 «L'analyse de la pratique : le cours des sens », Protée, n², pp. 9-19.

Foucault, Michel

1975 Surveiller et punir. Naissance de la prison, Paris, Gallimard.

1976 Histoire de la sexualité I. La volonté de savoir, Paris, Gallimard.

Grawitz, Madeleine

1991 Lexique des sciences sociales, Paris, Dalloz.

Greimas, Algirdas Julien

1976 « Pour une théorie des modalités », Langages, n43, pp. 90-107.

Jouët, Josiane

2000 « Retour critique sur la sociologie des usages », Réseaux, 100, vol. 18, pp.487-521.

Mucchielli, Alex

1997 «Le système des interactions et l'analyse du pouvoir dans les organisations : compréhension et explication du pouvoir », Quaderni, $\mathrm{n}^{\circ} 33$, pp. 19-44.

Rey, Alain (dir.)

2017 Le Petit Robert : dictionnaire alphabétique et analogique de la langue française, Paris, le Robert.

Stiegler, Bernard

2004 "Quand s'usent les usages », Azimuts, n 24.

Vial, Stéphane

2014 « De la spécificité du projet en design : une démonstration », Communication \& Organisation, $\mathrm{n}^{\circ} 46$, pp. 17-32. 